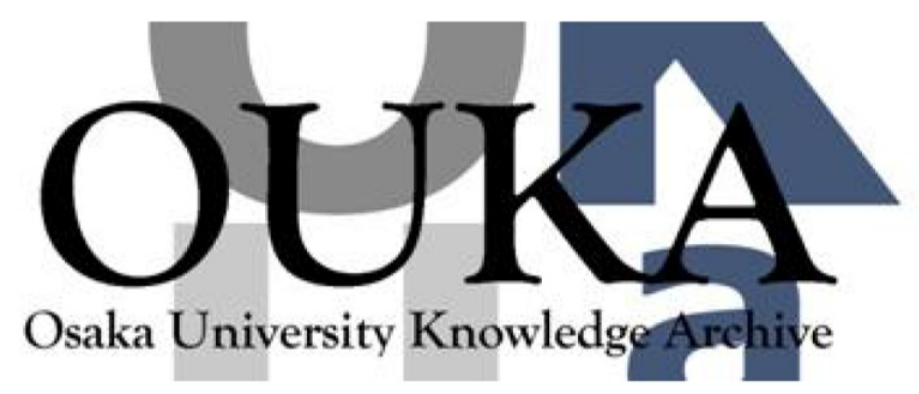

\begin{tabular}{|c|l|}
\hline Title & $\begin{array}{l}\text { Novel and Simple Approach to Estimating the } \\
\text { Actual Incidence of Blood and Body Fluid } \\
\text { Exposure }\end{array}$ \\
\hline Author(s) & $\begin{array}{l}\text { Yoshioka, Nori; Deguchi, Matsuo; Hagiya, } \\
\text { Hideharu et al. }\end{array}$ \\
\hline Citation & Clinical Laboratory. 63 p. 1481-p. 1486 \\
\hline Issue Date & $2017-10$ \\
\hline oaire:version & VoR \\
\hline URL & https://hdl. handle.net/11094/66991 \\
\hline rights & \\
\hline Note & \\
\hline
\end{tabular}

Osaka University Knowledge Archive : OUKA

https://ir. Library. osaka-u. ac. jp/

Osaka University 


\title{
Novel and Simple Approach to Estimating the Actual Incidence of Blood and Body Fluid Exposure
}

\author{
Nori Yoshioka ${ }^{1}$, Matsuo Deguchi ${ }^{2}$, Hideharu Hagiya ${ }^{3}$, Masanori Kagita ${ }^{4}$, Satomi Yukawa ${ }^{5}$, \\ Hiroko Tsukamoto $^{4}$, Hisao Yoshida ${ }^{3}$, Norihisa Yamamoto ${ }^{3}$, Yukihiro Akeda ${ }^{3}$, Yoshiko Nabetani ${ }^{6}$, \\ Ikuhiro Maeda $^{4}$, Yoh Hidaka ${ }^{4}$, Kazunori Tomono ${ }^{3}$ \\ ${ }^{1}$ Division of Infection Control and Prevention/Laboratory for Clinical Investigation, Osaka University Hospital, Japan \\ ${ }^{2}$ Laboratory for Clinical Investigation/Infection Control and Prevention, Osaka University Hospital, Japan \\ ${ }^{3}$ Division of Infection Control and Prevention, Osaka University Hospital, Japan \\ ${ }^{4}$ Laboratory for Clinical Investigation, Osaka University Hospital, Japan \\ ${ }^{5}$ National Hospital Organization Osaka National Hospital/Division of Infection Control and Prevention, Osaka University Hospital, Japan \\ ${ }^{6}$ Division of Infection Control and Prevention/Nursing Department, Osaka University Hospital, Japan
}

\begin{abstract}
SUMMARY
Background: There is no current way to determine the actual blood and body fluid exposure (BBFE) incidence in hospitals. We propose a simple, reliable, and widely available method for the accurate estimation of BBFE. Methods: Data for BBFE for healthcare workers between 2006 and 2015 at Osaka University Hospital were retrospectively extracted from the electronic records. Annual positivity of hepatitis $\mathrm{C}$ virus (HCV) antibody in the source individuals and overall patient population were calculated over time. We created an estimation formula focusing on the difference in HCV positivity between the source individuals and overall patient population for the actual number of BBFEs. A linear regression model was used to evaluate the temporal change in the reported and estimated BBFEs.

Results: During the study period, 937 BBFEs were reported. HCV positivity between the post-BBFE cohort and overall patient population greatly differed; the incidence ratio ranged from 2.1 to 5.7. The linear regression model revealed that the reported BBFEs did not significantly change during the study period (the slope, 1.315 [95\% confidence interval (C.I.): $\mathbf{- 0 . 8 4 9}$ to $3.480, p=0.199]$ ). The annual incidence ratio of the estimated and reported BBFEs significantly reduced over time (the slope, $-\mathbf{0 . 2 8 7}[95 \%$ C.I.: $-\mathbf{0 . 4 8 8}$ to $-0.086, p=0.011]$ ), indicating that, although the reported number of BBFEs seemed unchanged, the estimated incidence decreased.

Conclusions: We propose a novel and simple approach to estimating the actual incidence of BBFEs in hospitals using the difference in HCV positivity between the post-BBFE cohort and overall patient population.
\end{abstract}

(Clin. Lab. 2017;63:1481-1486. DOI: 10.7754/Clin.Lab.2017.170340)

Correspondence:

Nori Yoshioka, PhD

Division of Infection Control and Prevention

Osaka University Hospital, Japan

2-15 Yamadaoka

Suita, 565-0871 Osaka

Japan

Phone: +81 6-6879-5093

Fax: $\quad+81$ 6-6879-5094

Email: yoshioka@hp-infect.med.osaka-u.ac.jp

\section{KEY WORDS}

blood and body fluid exposure, healthcare worker, hepatitis $\mathrm{C}$ virus, occupational infection control prevention

\section{INTRODUCTION}

Blood and body fluid exposure (BBFE) during daily hospital routines is inevitable for health-care workers (HCWs) [1], despite the development of safety-engineered devices and accumulated knowledge for preven- 
tion [2]. According to the World Health Organization, the annual global incidence of percutaneous BBFE in $\mathrm{HCWs}$ is as high as 3 million [3]. Although the major concerns as a consequence of BBFE are hepatitis B virus (HBV), hepatitis $\mathrm{C}$ virus (HCV), and human immunodeficiency virus (HIV) infections [4], more than 50 blood-borne pathogens can cause infections after BBFE [5].

Based on the US General Accounting Office, follow-up expenses, excluding treatment, for HCWs after BBFE are $>\$ 500$ per incident [6]. In addition to the financial issues, occupational exposure results in emotional distress. Thus, a systematic approach for the prevention of BBFE is expected in hospitals.

Comprehension of the true incidence of BBFE is of great importance for infection control practitioners to evaluate their activities for the prevention of occupational exposure and to develop appropriate preventive strategies. Although HCWs are generally supposed to report a BBFE incidence to infection control services, the number of reported BBFE is almost without exception less than that of true events [7]. Namely, it is almost impossible to determine the actual number of BBFEs because of under-reporting [5,8-11]. For example, more than $80 \%$ of syringe injuries were unreported in Taiwan before 2000 [12,13]. Understandably, the reported BBFEs may only be the tip of the iceberg, and a decrease in the number of reported BBFEs does not accurately reflect a true reduction in the hospital. No gold standard to estimate the actual incidence of BBFE exists, and all previous interventional or observational studies concerning BBFEs have been merely based on the reported number, which could under-represent the actual incidence [14-16].

In this study, we propose a novel, simple, and widely available estimation formula that uses HCV positivity in a post-BBFE cohort and the overall patient population in hospitals. Using this approach, we estimated the actual incidences of BBFEs in our university hospital for the last 10 years and evaluated the difference between the reported and estimated numbers of BBFEs.

\section{MATERIALS AND METHODS}

This study was conducted at Osaka University Hospital, a 1086-bed tertiary care academic facility in Japan. Data, including the nature of the injury and occupation, for HCWs with BBFE between 2006 and 2015 were extracted from the electronic database of the Infection Control Service and Clinical Laboratory. All BBFEs were registered in the Exposure Prevention Information Network, Japan. The study protocol was approved by the Institutional Review Board of Osaka University Hospital (No. 10105-2). The need for informed consent was waived because we retrospectively collected data without the use of any individually identifying information or the application of any intervention.
In our hospital, we measure seropositivity of the source individuals for HBV (antigen/antibody), HCV (antibody), HIV (antigen/antibody), human T-cell leukemia virus type 1/2 (antibody), and syphilis (rapid plasma reagin) using blood samples submitted immediately after BBFE events to clearly determine the infectious profile at the time of exposure. Of these, we collected data for $\mathrm{HCV}$ positivity in the source individuals. Also, the annual HCV positivity of the overall patient population at our facility was calculated. If HCV antibody was measured $\geq 2$ times within a year for an individual, only one of the measurements was included. The HCV antibody was measured using the Architect i2000 Analyzer (Abbott Japan Co., Ltd Tokyo, JAPAN).

Our estimation formula consists as follows;

$i$ the number of reported BBFEs

ii the number of reported BBFEs by an unknown source

iii HCV positivity of source individuals in the postBBFE cohort, and

iv $\mathrm{HCV}$ positivity in the overall patient population

Estimated incidence of BBFEs $=[(\mathrm{i})-(\mathrm{ii})] \times \frac{\text { (iii) }}{(\mathrm{iv})}+($ (ii)

We focused on the difference in HCV positivity between the post-BBFE cohort and overall patient population. Theoretically, a BBFE occurs randomly regardless of $\mathrm{HCV}$ positivity, and the positive rate in the postBBFE cohort should be comparable with that in the overall patient population. However, the HCV positivity of the source individuals in the post-BBFE cohort was much higher than that in the overall patient population, potentially because of the under-reporting of BBFE events. For instance, when a HCW experiences a BBFE with an HCV-positive source individual, he or she presumably reports the exposure to the Infection Control Service. However, with an HCV-negative source, the incidence may not be reported. With fewer reports of BBFEs with $\mathrm{HCV}$-negative sources, the $\mathrm{HCV}$ positivity in the post-BBFE cohort would be higher. Thus, in the estimation formula, we calculated the ratio of $\mathrm{HCV}$ positivity in the post-BBFE cohort to that of the overall patient population and multiplied it by the number of source-apparent BBFEs.

Statistical analysis was performed using EZR software, which is a modified version of $\mathrm{R}$ Commander (version 2.2-5) based on $\mathrm{R}$ (version 3.3.1) [17]. We defined the annual incidence ratio as the ratio of the estimated BBFEs to the reported BBFEs in a year. We applied a linear regression line of this ratio to evaluate the difference in the annual incidence between the reported and estimated BBFEs over time. Statistical significance was calculated by comparing the regression line to a zero line. A p-value $<0.05$ was considered statistically significant. 
Table 1. Incidences of blood and body fluid exposure (BBFE) at Osaka University Hospital, 2006 to 2015.

\begin{tabular}{|c|c|c|c|c|c|c|c|c|c|c|c|}
\hline & 2006 & 2007 & 2008 & 2009 & 2010 & 2011 & 2012 & 2013 & 2014 & 2015 & Total \\
\hline BBFE incidence & 83 & 102 & 94 & 83 & 101 & 82 & 102 & 105 & 87 & 98 & 937 \\
\hline \multicolumn{12}{|c|}{ Nature of injury } \\
\hline Needles & 69 & 90 & 76 & 66 & 864 & 71 & 80 & 73 & 60 & 69 & 740 \\
\hline Sharps & 3 & 6 & 3 & 5 & 3 & 1 & 5 & 11 & 9 & 8 & 54 \\
\hline Mucocutaneous exposure & 3 & 2 & 8 & 4 & 6 & 5 & 14 & 11 & 10 & 13 & 76 \\
\hline Unknown & 3 & 2 & 5 & 4 & 1 & 3 & 1 & 1 & 1 & 2 & 23 \\
\hline Others * & 5 & 2 & 2 & 4 & 5 & 2 & 2 & 9 & 7 & 6 & 44 \\
\hline \multicolumn{12}{|c|}{ Occupation } \\
\hline Medical doctor & 51 & 42 & 39 & 28 & 47 & 29 & 29 & 38 & 38 & 47 & 388 \\
\hline Nurse & 26 & 54 & 48 & 46 & 40 & 44 & 62 & 56 & 41 & 39 & 456 \\
\hline Laboratory technician & 2 & 2 & $\mathbf{0}$ & 3 & 4 & 3 & 5 & 5 & 2 & 5 & 31 \\
\hline Service staff & 1 & 3 & 3 & 5 & 7 & 3 & 1 & 4 & 4 & 4 & 35 \\
\hline Others $^{* *}$ & 3 & 1 & 4 & 1 & 3 & 3 & 5 & 2 & 2 & 3 & 27 \\
\hline
\end{tabular}

" - Forceps, glass ampoules, wires, etc., ${ }^{* *}$ - Nurse's aides, students, patients, and unknown cases.

Table 2. Annual Hepatitis C virus (HCV) positivity in the post-blood and body fluid exposure (BBFE) cohort and overall patient population.

\begin{tabular}{|c|c|c|c|c|c|c|c|c|c|c|}
\hline & 2006 & 2007 & 2008 & 2009 & 2010 & 2011 & 2012 & 2013 & 2014 & 2015 \\
\hline $\begin{array}{c}\text { Post-BBFE } \\
\text { cohort }\end{array}$ & $\begin{array}{c}35.1 \% \\
(81)\end{array}$ & $\begin{array}{c}27.2 \% \\
(94) \\
\end{array}$ & $\begin{array}{c}36.1 \% \\
(91) \\
\end{array}$ & $\begin{array}{c}22.4 \% \\
(82) \\
\end{array}$ & $\begin{array}{c}17.4 \% \\
(97)\end{array}$ & $\begin{array}{c}22.9 \% \\
(80)\end{array}$ & $\begin{array}{c}20.7 \% \\
(102)\end{array}$ & $\begin{array}{c}18.2 \% \\
(105)\end{array}$ & $\begin{array}{c}\mathbf{1 3 . 8 \%} \\
(87) \\
\end{array}$ & $\begin{array}{c}10.2 \% \\
(98)\end{array}$ \\
\hline $\begin{array}{c}\text { Overall } \\
\text { patient } \\
\text { population }\end{array}$ & $\begin{array}{c}6.0 \% \\
(11821)\end{array}$ & $\begin{array}{c}5.7 \% \\
(12071)\end{array}$ & $\begin{array}{c}5.9 \% \\
(12260)\end{array}$ & $\begin{array}{c}4.9 \% \\
(11786)\end{array}$ & $\begin{array}{c}5.4 \% \\
(11822)\end{array}$ & $\begin{array}{c}4.8 \% \\
(12429)\end{array}$ & $\begin{array}{c}4.4 \% \\
(12326)\end{array}$ & $\begin{array}{c}4.4 \% \\
(13119)\end{array}$ & $\begin{array}{c}4.5 \% \\
(13170)\end{array}$ & $\begin{array}{c}4.6 \% \\
(14180)\end{array}$ \\
\hline
\end{tabular}

Overall patient population includes both in- and outpatients. The annual numbers of cases in which HCV antibody were measured are shown in parenthesis.

\section{RESULTS}

During the study period, a total of 937 BBFEs were reported to the Infection Control Service, including 740 needle injuries (79.3\%), 54 sharp injuries (5.7\%), 76 mucocutaneous exposures $(80 \%), 23$ unknown events $(2.6 \%)$, and 44 others $(4.7 \%)$. By occupation, medical doctors $(388,41.7 \%)$, nurses $(456,48.6 \%)$, laboratory technicians $(31,3.3 \%)$, service staff $(35,3.8 \%)$, and others $(27,2.9 \%)$ were affected (Table 1$)$. HCV antibody was examined in 917 of the 937 BBFE events (97.9\%). The annual HCV positivity between the postBBFE cohort (10.2 - 35.1\%) and overall patient population (4.4 - 6.0\%) greatly differed (Table 2$)$.

Figure 1 shows the numbers of reported and estimated BBFEs in each year using the estimation formula, as well as their ratios over time. The linear regression model revealed that the reported number of BBFEs did not change significantly during the study period (the slope, 1.315 [95\% confidence interval (C.I.): -0.849 to $3.480, p=0.199])$. However, the annual incidence ratio declined significantly (the slope, -0.287 [95\% C.I.: -0.488 to $-0.086, \mathrm{p}=0.011])$.

\section{DISCUSSION}

In this study, we proposed a novel estimation formula to determine the actual number of BBFEs by focusing on the difference in $\mathrm{HCV}$ positivity between the source individuals in a post-BBFE cohort and the overall patient population. $\mathrm{HCV}$ is an RNA mono-stranded enveloped virus that has a wide distribution, infecting $2-3 \%$ of the world population [18]. For instance, in Japan, the $\mathrm{HCV}$ prevalence in the general population is reportedly $1.0-1.9 \%$ [19]. In the United States, 2.7 million per- 


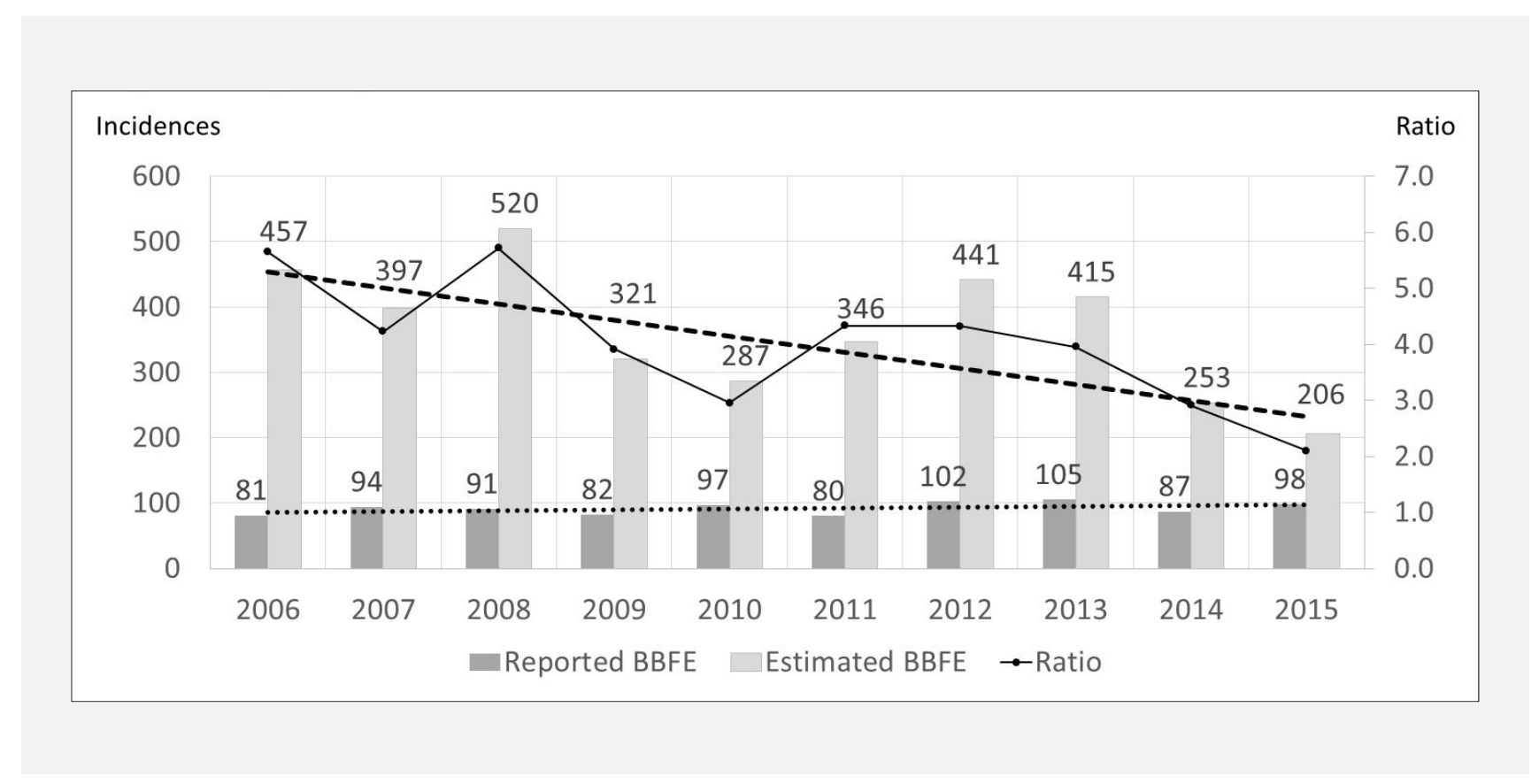

Figure 1. Annual reported and estimated blood and body fluid exposure (BBFE) incidents and the annual incidence ratio.

A solid line indicates the ratio of estimated BBFEs to reported BBFEs (annual incidence ratios). Bold and fine dotted lines are linear regression lines of the annual incidence ratio and reported number of BBFEs, respectively. The annual incidence ratio decreased significantly over time $(p=0.011)$, while the reported number of BBFEs did not.

sons, accounting for approximately $0.9 \%$ of the total population, have chronic HCV infection [20]. Thus, our equation provides a widely available method for infection control practitioners to practically estimate the actual incidence of BBFEs in their facilities.

Implementation of effective infection control strategies can reduce the transmission risk of viral hepatitis and HIV [21], and many preventable measures for BBFEs have been developed. However, BBFEs might not be reported properly; among the various occupations in hospitals, for instance, medical doctors are less likely to report BBFEs to the responsible sections than other HCWs [22]. Thus, poor reporting systems may provide incorrect estimates. The result of statistical analysis showed that the reported number of BBFEs remained at a constant level, while the estimated incidence tended to decline over time at our facility. These results can support the effectiveness of preventive measures for BBFEs in our hospital and the ability of our simple approach to offer an objective evaluation of the actual incidence of BBFEs.

The strength of this study is the accurate data for HCV positivity in the source individuals. Because of additional costs, previous data for infectious profiles have been applied for post-BBFE evaluation in many hospitals, although past data do not necessarily reflect the current status. For more than 10 years, we have required the known source individuals to submit their blood sam- ples following BBFE events to understand their infectious profiles. Therefore, the HCV positivity of the present post-BBFE cohort is reliable.

$\mathrm{HBV}$ cannot replace $\mathrm{HCV}$ in the estimation formula. Although the HBV vaccination policy may differ in each medical facility or country, the proportion of HCWs vaccinated for $\mathrm{HBV}$ is higher than that in the general population. Thus, measurement of HB antibody is not useful for the estimation. Data for other HBV-related antigens or antibodies are usually not as frequently measured in the overall patient population and cannot provide a reliable adjustment. In contrast to HBV, vaccination for HCV has not been established [23], and this disadvantage turned out to be a strength for our method. The prevalence of HIV is still lower in Japan than in other countries, and its positivity is not applicable for the estimation. However, it may be possible to apply HIV positivity to our estimation formula in particular regions where HIV prevalence is comparatively high. Compared to the general population [19], HCV positivity at our facility was relatively higher, ranging from $4.4 \%$ to $6.0 \%$. Hospitalized and ambulatory patients are usually older than the general population. Also, patients with $\mathrm{HCV}$ infection have been referred to our hospital because of its provision of tertiary care.

Several limitations of this study should be mentioned. First, the absence of a gold standard reference for the actual BBFE incidence should be noted. However, no 
matter how much time passes, it is impossible to determine the actual number because of under-reporting. Second, the implementation rates of HCV serology among the overall patient population may influence the accuracy of the estimation. Less measured in the overall population, the HCV positivity, which is a denominator in the estimation formula, can vary. In Japan, HCV antibody is generally tested when patients are hospitalized or undergo surgeries. Therefore, we consider the HCV positivity of overall patient population in this study is comparatively reliable. Third, the nature of a single facility study might affect the generalizability of our methodology. Also, comparison of the estimated number of BBFEs among medical facilities may be difficult because of the different hospital characteristics.

\section{CONCLUSION}

We proposed a new, simple estimation formula for the estimation of the actual incidence of BBFEs using the difference in HCV positivity between the post-BBFE cohort and overall patient population. The reported number does not necessarily reflect the true incidence of BBFEs in each medical facility, while our method can provide a reliable estimation. By following the trend in the estimated number, it is possible to better comprehend and evaluate the real occurrence of BBFEs at each facility. In our facility, although the reported incidence of BBFEs did not change, the estimated incidence significantly decreased during the study period. Under-reporting of BBFEs continues to persist. A better understanding of BBFE occurrence in each medical facility can lead to appropriate evaluations and countermeasures for preventable BBFE.

\section{Authors' Contributions:}

Study conception and design: M. Deguchi and N. Yoshioka. Acquisition of data: M. Kagita and H. Tsukamoto. Analysis and interpretation of data: N. Yoshioka, M. Deguchi, H. Hagiya, and S. Yukawa. Drafting of manuscript: H. Hagiya and N. Yoshioka. Critical revision: N. Yamamoto, H. Yoshida, Y. Akeda, Y. Nabetani, I. Maeda, Y. Hidaka, and K. Tomono.

\section{Acknowledgement:}

We would like to thank all the staff belonging to the Infection Immunity, Division of Clinical Investigation, Osaka University Hospital, for their contribution to the data collection. Also, we appreciate the useful advice for statistical analysis from Dr. Koji Yamamoto (Department of Clinical Epidemiology and Biostatistics, Graduate School of Medicine, Osaka University). Last, we are grateful to all the personnel contributing to the infection control activities in our hospital.

\section{Declaration of Interest:}

The authors declare that they have no competing interests.

\section{References:}

1. Sepkowitz KA. Occupationally acquired infections in health care workers: part II. Ann Intern Med 1996;125:917-28 (PMID: 8967 673).

2. Kanamori H, Weber DJ, DiBiase LM, et al. Impact of Safety-Engineered Devices on the Incidence of Occupational Blood and Body Fluid Exposures Among Healthcare Personnel in an Academic Facility, 2000-2014. Infect Control Hosp Epidemiol 2016;37: 497-504 (PMID: 26856246).

3. Prüss-Üstün A, Rapiti E, Hutin Y. Estimation of the global burden of disease attributable to contaminated sharps injuries among health-care workers. Am J Ind Med 2005;48:482-90 (PMID: 162 99710).

4. MacCannell T, Laramie AK, Gomaa A, Perz JF. Occupational exposure of health care personnel to hepatitis B and hepatitis C: prevention and surveillance strategies. Clin Liver Dis 2010;14:23-36 (PMID: 20123437).

5. Tarantola A, Abiteboul D, Rachline A. Infection risks following accidental exposure to blood or body fluids in health care workers: a review of pathogens transmitted in published cases. Am J Infect Control 2006;34:367-75 (PMID: 16877106).

6. Phillips EK, Conaway M, Parker G, Perry J, Jagger J. Issues in understanding the impact of the Needlestick Safety and Prevention Act on hospital sharps injuries. Infect Control Hosp Epidemiol 2013;34:935-9 (PMID: 23917907).

7. Osborn EH, Papadakis MA, Gerberding JL. Occupational exposures to body fluids among medical students: a seven-year longitudinal study. Ann Intern Med 1999;130:45-51 (PMID: 9890850).

8. Schmid K, Schwager C, Drexler H. Needlestick injuries and other occupational exposures to body fluids amongst employees and medical students of a German university: incidence and followup. J Hosp Infect 2007;65:124-30 (PMID: 17174445).

9. Au E, Gossage J, Bailey S. The reporting of needlestick injuries sustained in theatre by surgeons: are we under-reporting? J Hosp Infect 2008;70:66-70 (PMID: 18602192).

10. Haiduven D, Simpkins S, Phillips E, Stevens D. A survey of percutaneous/mucocutaneous injury reporting in a public teaching hospital. J Hosp Infect 1999;41:151-4 (PMID: 10063478).

11. Voide C, Darling K, Kenfak-Foguena A, Erard V, Cavassini M, Lazor-Blanchet C. Underreporting of needlestick and sharps injuries among healthcare workers in a Swiss University Hospital. Swiss Med Wkly 2012;142:w13523 (PMID: 22328010).

12. Guo Y, Shiao J, Chuang YC, Huang KY. Needlestick and sharps injuries among health-care workers in Taiwan. Epidemiol Infect 1999;122:259-65 (PMID: 10355790).

13. Shiao JS, McLaws ML, Huang KY, Ko WC, Guo YL. Prevalence of nonreporting behavior of sharps injuries in Taiwanese health care workers. Am J Infect Control 1999;27:254-7 (PMID: 10358 228).

14. Tarigan LH, Cifuentes M, Quinn M, Kriebel D. Prevention of needle-stick injuries in healthcare facilities: A meta-analysis. Infect Control Hosp Epidemiol 2015;36:823-9 (PMID: 25765502). 
15. Nouetchognou JS, Ateudjieu J, Jemea B, Mbanya D. Accidental exposures to blood and body fluids among health care workers in a Referral Hospital of Cameroon. BMC Res Notes 2016;9:1 (PMID: 26879949).

16. Myers DJ, Lipscomb HJ, Epling C, et al. Surgical Team Stability and Risk of Sharps-Related Blood and Body Fluid Exposures During Surgical Procedures. Infect Control Hosp Epidemiol 2016;37:512-8 (PMID: 26856378).

17. Kanda Y. Investigation of the freely available easy-to-use software 'EZR' for medical statistics. Bone Marrow Transplant 2013; 48:452-8 (PMID: 23208313).

18. Lozano R, Naghavi M, Foreman K, et al. Global and regional mortality from 235 causes of death for 20 age groups in 1990 and 2010: a systematic analysis for the Global Burden of Disease Study 2010. Lancet 2013;380:2095-128 (PMID: 23245604).

19. Sievert W, Altraif I, Razavi HA, et al. A systematic review of hepatitis $\mathrm{C}$ virus epidemiology in Asia, Australia and Egypt. Liver Int 2011;31:61-80 (PMID: 21651703).

20. Denniston MM, Jiles RB, Drobeniuc J, et al. Chronic hepatitis C virus infection in the United States, national health and nutrition examination survey 2003 to 2010. Ann Intern Med 2014;160:293300 (PMID: 24737271).

21. Gupta A, Anand S, Sastry J, et al. High risk for occupational exposure to HIV and utilization of post-exposure prophylaxis in a teaching hospital in Pune, India. BMC Infect Dis 2008;8:1 (PMID: 18939992).

22. Marković-Denić L, Branković M, Maksimović N, et al. Occupational exposures to blood and body fluids among health care workers at university hospitals. Srp Arh Celok Lek 2013;141: 789-93 (PMID: 24502099).

23. Honegger JR, Zhou Y, Walker CM. Will there be a vaccine to prevent HCV infection? Semin Liver Dis 2014;34:79-88 (PMID: 24782261). 